\title{
Stripping Away the Soil: Plant Growth Promoting Microbiology Opportunities in Aquaponics
}

\begin{abstract}
Ryan P. Bartelme', Ben O. Oyserman ${ }^{2,3}$, Jesse E. Blom ${ }^{4}$, Osvaldo J. Sepulveda-Villet ${ }^{1}$ and Ryan J. Newton ${ }^{1 *}$

${ }^{1}$ School of Freshwater Sciences, University of Wisconsin-Milwaukee, Milwaukee, WI, United States, ${ }^{2}$ Bioinformatics Group, Wageningen University \& Research, Wageningen, Netherlands, ${ }^{3}$ Department of Microbial Ecology, Netherlands Institute of Ecology, Wageningen, Netherlands, ${ }^{4}$ Johns Hopkins Center for a Livable Future, Department of Environmental Health and Engineering, Bloomberg School of Public Health, Johns Hopkins University, Baltimore, MD, United States
\end{abstract}

As the processes facilitated by plant growth promoting microorganisms (PGPMs) become better characterized, it is evident that PGPMs may be critical for successful sustainable agricultural practices. Microbes enrich plant growth through various mechanisms, such as enhancing resistance to disease and drought, producing beneficial molecules, and supplying nutrients and trace metals to the plant rhizosphere.

OPEN ACCESS

Edited by: Jaime Romero, Universidad de Chile, Chile

Reviewed by:

David Dowling,

Institute of Technology Carlow, Ireland

Usman Irshad,

COMSATS Institute of Information Technology, Pakistan

${ }^{*}$ Correspondence:

Ryan J. Newton newtonr@uwm.edu

Specialty section: This article was submitted to Aquatic Microbiology, a section of the journal

Frontiers in Microbiology

Received: 25 September 2017 Accepted: 04 January 2018

Published: 22 January 2018

Citation:

Bartelme RP, Oyserman BO, Blom JE, Sepulveda-Villet OJ and Newton RJ (2018) Stripping Away the Soil: Plant Growth Promoting

Microbiology Opportunities in Aquaponics. Front. Microbiol. 9:8. doi: 10.3389/fmicb.2018.00008
Previous studies of PGPMs have focused primarily on soil-based crops. In contrast, aquaponics is a water-based agricultural system, in which production relies upon internal nutrient recycling to co-cultivate plants with fish. This arrangement has management benefits compared to soil-based agriculture, as system components may be designed to directly harness microbial processes that make nutrients bioavailable to plants in downstream components. However, aquaponic systems also present unique management challenges. Microbes may compete with plants for certain micronutrients, such as iron, which makes exogenous supplementation necessary, adding production cost and process complexity, and limiting profitability and system sustainability. Research on PGPMs in aquaponic systems currently lags behind traditional agricultural systems, however, it is clear that certain parallels in nutrient use and plant-microbe interactions are retained from soil-based agricultural systems.

Keywords: aquaponics, plant growth promoting microorganisms, recirculating aquaculture, chlorosis, rhizosphere, microbiome

\section{AQUAPONICS - STRIPPING AWAY THE SOIL}

Aquaponics, the combined culture of fish and plants in recirculating water systems was pioneered in the 1970s (Sneed et al., 1975; Naegel, 1977; Lewis et al., 1978) as an environmentally sustainable agricultural method based on the concepts of minimal water use and minimal impact on environmental water quality compared to traditional agricultural methods (Blidariu and Grozea, 2011). In addition to producing salable crops, aquaponics is valued for its positive development of community and economic opportunity in urban areas (Goodman, 2011), and its wide-ranging educational benefits for students through the post-secondary level (Hart et al., 2014; Genello et al., 2015). Despite these benefits, microbial research supporting aquaponic crop production lags 
behind traditional agricultural systems. Here, we present the case that aquaponic systems provide relatively untapped potential for research on plant-microbe interactions.

Aquaponics is a highly engineered agricultural system that uses fish effluent (which comprises both particulate waste solids and dissolved nutrients) from a recirculating aquaculture subsystem as nutrient medium to grow edible plants in attached hydroponic subsystems. In return, nutrient removal through plant absorption and growth, parallel to their associated microbiota, decreases dissolved solid and ionic concentrations, which, in turn, benefits fish production by improving overall water quality parameters, including the removal of toxic metabolites, such as ammonia and nitrite. Together, this circular and beneficial relationship between fish, plants, and microbes reduces water usage compared to traditional agriculture. Historically, aquaponics research was driven by the recirculating aquaculture community; so most technological advancement was focused on optimizing water quality for fish production purposes (Sneed et al., 1975; Naegel, 1977; Lewis et al., 1978). However, recent energy analyses and industry surveys of aquaponic systems concluded that profitability is greater when a plant-centric production approach is adopted (Love et al., 2015a,b). Incorporation and testing of hydroponic method compatibility is therefore critical to integrating simultaneous fish and plant production. For example, using a hydroponic-centered production setup, Schmautz et al. (2016) found that the aquaculture-subsystem provided the necessary nutrients to produce cherry tomatoes with nutrient content that exceeded those available at local markets. However, to improve plant growth in plant-centric aquaponic systems, micronutrient supplementation (e.g., iron, calcium, and potassium) is often required (Rakocy et al., 2004; Roosta, 2014; Bittsanszky et al., 2016). Additionally, chemical inputs are needed to counteract disease and other plant stressors (Nguyen et al., 2016), which adds long-term operational expenses (Love et al., 2015a). Thus, despite the benefits of these integrated agricultural systems, high capital expenses during system construction (Engle, 2015), and the abovementioned chemical supplementation make achieving economic sustainability a challenge. Continued research on micronutrient transport and transformation between aquaponic subsystems is needed to identify shortcomings and optimize engineering paradigms in aquaponic systems.

Plant growth promoting microorganisms (PGPMs) may be an effective alternative to chemical inputs for dealing with plant growth requirements and stressors in aquaponic systems. Plants recruit PGPMs from the surrounding environment to their rhizosphere using specific chemical signaling (DeVries and Wallenstein, 2017). For example, under phosphorus (P)-limiting conditions, the plant hormone strigolactone is released into the rhizosphere, where it serves as a signaling molecule to initiate associations with fungi (Akiyama et al., 2005). In soil-based environments PGPMs are known to enhance plant growth via a number of mechanisms, including: nitrogen fixation, organic matter mineralization, root growth promotion, protection against pathogens, and increasing the bioavailability of nutrients, including micronutrients such as iron
(Höflich et al., 1995; Loper and Henkels, 1997, 1999; Mendes et al., 2011; Loper et al., 2012; Malusá et al., 2012; Marasco et al., 2012; Rashid et al., 2012; Coleman-Derr and Tringe, 2014; Dias et al., 2014; Pii et al., 2015; da Silva Cerozi and Fitzsimmons, 2016; Khalifa et al., 2016). In soilless environments, PGPM research is limited, but existing studies suggest PGPMs also play a significant role in plant growth and health (Gravel et al., 2006; Villarroel et al., 2011; da Silva Cerozi and Fitzsimmons, 2016; Nguyen et al., 2016; Sheridan et al., 2016).

Regardless of the agricultural system, root health is essential to the survival of plants; so one focus area for aquaponic PGPM research should be microbial root colonization. Arbuscular mycorrhizal fungi are well-documented plant growth promoting fungi that colonize plant roots. In traditional soil-based agriculture, arbuscular mycorrhizal fungi promote phosphorus uptake and enhance biomass production (Govindasamy et al., 2011). Arbuscular mycorrhizal fungi also appear to be important for plant health in hydroponics. For example, in one hydroponic system study, arbuscular mycorrhizal fungi inhibited Fusarium spp. from inducing root rot in tomatoes grown under near-commercial conditions (Utkhede, 2006). While arbuscular mycorrhizal fungi are a commonly cited PGPM, many different microorganisms are thought to be PGPMs. One such group, rhizobia, were discovered in the 19th Century (Beijerinck, 1888), and now these diazotrophic bacteria are recognized as essential agents in promoting growth among crops such as legumes, rice, and wheat (Govindasamy et al., 2011; Ji et al., 2014; Majeed et al., 2015). Interestingly, iron siderophores facilitate the formation of rhizobium diazotrophic nodules (Barton et al., 1996; Brear et al., 2013), suggesting micronutrients may play a role in PGPM colonization in other agricultural systems, such as hydroponics/aquaponics. However, some PGPM benefits may come at an adaptive cost, such as increased sensitivity to insect herbivory (Barazani and Baldwin, 2013), but controlled environmental agriculture can account for invertebrate pest problems (Fox et al., 2012). Ultimately, research on PGPM in aquaponic systems may alleviate costly nutrient supplementation by properly integrating PGPM driven environmental processes into system design.

In addition to benefitting aquaponic crop production, PGPM research in soilless engineered environments has the potential to advance the fundamental understanding of rhizosphere microorganism associations. It is clear plants recruit PGPMs to their rhizosphere, but the mechanisms driving plant growth promoting rhizosphere interactions are difficult to disinter from soil-based studies (DeVries and Wallenstein, 2017). Soil matrices are chemically complex and heterogeneous, exhibiting immensely diverse microbial communities. Additionally, given the large variability among soil and crop types (DeVries and Wallenstein, 2017), rhizosphere recruitment of PGPMs in this environment remains mainly theoretical and often limited to a case-by-case basis. The complexity of the soil matrix also adds technological hurdles to studying PGPMs. The soil matrix often hinders nucleic acid extraction and, subsequently, sequence-based analyses of microorganisms, thus 
inhibiting the exploration of microbial community structure (Carini et al., 2016), genetic signatures pertaining to nutrient processing (Krsek and Wellington, 1999; Martin-Laurent et al., 2001), and the identification of microbial guilds to utilize in rhizosphere engineering (Savka et al., 2013; Mueller and Sachs, 2015; Pii et al., 2015; Dessaux et al., 2016). In contrast to soil-based agricultural systems, aquaponic systems operate in highly monitored and controlled environments (e.g., pH, temperature, hydraulic retention time, nutrient concentrations, etc.), and lack the confounding variability and complexity of the soil-matrix. As a result, these systems represent, scalable, highly reproducible, and adjustable laboratories for PGPM research, where discoveries made in a research setting may be more directly transferred to an industrial or practical application.

\section{THE CHALLENGES OF INTEGRATED SYSTEM DESIGN IN AQUAPONICS}

Aquaponic systems must balance the physiological requirements of both plant and fish in order to maintain their health. This balance makes even basic system design challenging, such as identifying plant and fish species that are compatible. The integration of distinct fish and plant subsystems also means operational change in any one component inherently impacts all other components, thereby creating a fairly high level of ecological complexity. For example, solid waste in aquaponic systems primarily consists of fish feed and feces, which, when decomposed, act as fertilizer for the hydroponic subsystem. Calculated fish feed rates relate to plant grow-bed size, but feed conversion and nutrient assimilation varies with feed protein type (i.e., plant-derived vs. teleost protein extracts) and plant crop-type (Rakocy et al., 2006; Timmons and Ebeling, 2013; Hu et al., 2015; Medina et al., 2016). Excess solid waste increases oxygen demand leading to hypoxic conditions in the rhizosphere, and may generate toxic concentrations of ammonia and nitrite (Rakocy, 2012; Danaher et al., 2013). Therefore, proper solids management is necessary to maintain the oxygen gradient around the plant roots allowing for colonization of PGPMs and preventing phytopathogen growth. However, in the roots of the hydroponic subsystem an appropriate level of solid waste re-mineralization is essential to supply micro- and macronutrients to the plants (Rakocy et al., 2012). Basic system design constraints influence overall microbial community structure and suggest microbial niche differentiation within system components, but the influence of environmental conditions has yet to be explored in aquaponic systems (Schmautz et al., 2017). Food web interactions, including predation on bacteria and archaea and nutrient or energy transfer from microbial eukaryotic activity, such as that from micro-fungi, may also confound system designs in yet unknown ways. These food web interactions have had little consideration in system designs to date, but deserve thorough analysis as control points for microbial-plant interactions and in experiments aimed at optimizing aquaponic system technology.

\section{IRON LIMITATION: A CASE STUDY FOR PGPM RESEARCH IN AQUAPONIC PLANT PRODUCTION}

Commonly, iron is supplemented in the hydroponic subsystems of aquaponics configurations; however, little attention has been paid to exactly why this supplementation is required. Herein we review what is known about iron requirements in aquaponics and discuss possible iron supplementation strategies that do not require industrially produced chelated iron. Iron is an essential molecule for a multitude of metalloprotein structures (e.g., hemoglobin, chlorophyll, and cytochromes), and therefore, demand is high from all biological components of an aquaponic system. Fish assimilate low amounts of iron relative to terrestrial livestock (van Dijk et al., 1975), and often fish iron needs are met or frequently exceeded, with commercial feeds (Watanabe et al., 1997), so little attention is paid to this component of aquaculture operations. In contrast, although undigested fish feed contains excess iron, plant grow beds in aquaponics are often limited by bioavailable iron $\left(\mathrm{Fe}^{2+}\right)$. This nutrient deficiency is a known cause of chlorosis in the hydroponic subsystem crops, but may not be evident in a system until vegetable products have been raised for multiple generations (Rakocy et al., 2004). In the University of the Virgin Islands system, chelated iron is added at a concentration of $2 \mathrm{mg} / \mathrm{L}$ per day to prevent chlorosis (Rakocy et al., 2004). One major factor driving iron deficiency is that soluble ferrous iron $(2+)$ easily crosses the rhizoplane of the roots, but ferric iron (3+) is insoluble. Consequently, the competing chemical reactions driving $\mathrm{Fe}^{2+}$ to $\mathrm{Fe}^{3+}$ (i.e., the speciation of iron in natural water systems by hydroxyl radicals and ionic interactions) and $\mathrm{pH}$ dependency complicate the mass balance of iron in aquaponic systems (Rose and Waite, 2002; Waite, 2002). Biotic factors in aquaponic systems may also reduce available iron for plants. Endemic microbial communities scavenge iron for constructing metalloprotein centers (Andrews et al., 2003), but in aquaponics the extent of this iron demand remains unknown.

Limitation of biologically available iron is a relatively common phenomenon across environments containing photosynthetic organisms. One such environment is the ocean, where primary productivity depends on soluble iron, though iron remains sequestered in microbial amphiphilic siderophores (Boiteau et al., 2016). Published literature on the role of siderophores in soil-based agriculture point to both an enhancement of growth and a link to pathogenesis (Kloepper et al., 1980; Neilands and Leong, 1986). Pseudomonas fluorescens Pf-5 is one PGPM known to increase the bioavailability of iron through siderophore production in iron deficient soils (Loper and Henkels, 1997). Interestingly, some pseudomonads, like Pseudomonas putida, are able to scavenge iron from other siderophores under laboratory conditions and promote iron uptake in experimental cucumber seedlings (Loper and Henkels, 1997). The "siderophore theft" may be indicative of pseudomonad PGPM's ability to remediate disease (Loper and Buyer, 1991; Loper and Henkels, 1997). Furthermore, genomic analyses of Pseudomonas spp. indicate a distinct ability 
to modulate the surrounding rhizosphere community through antifungal and bacteriocin production, in addition to siderophore production (Loper et al., 2012). In soil-based agriculture, other bacterial species such as, Bacillus and Paenibacillus spp. exhibit similar PGPM characteristics (Govindasamy et al., 2011). All of the aforementioned PGPM microbes found in soil studies may allow aquaponics practitioners to biologically remediate regularly occurring nutrient deficiencies. Since there is evidence PGPM ecophysiologies work to overcome micronutrient deficiencies in engineered environments (Villarroel et al., 2011), there is ample opportunity to research their usefulness for aquaponic system design and management.

All nutrient rich hydroponic systems, including aquaponics, must balance the promotion of beneficial microorganisms while minimizing the growth and rapid spread of plant pathogens (Lee and Lee, 2015). Though the resiliency of aquaponic systems to phytopathogen infection requires experimental study, other groups of PGPM's (such as Bacillus spp. and Paenibacillus spp.) may be linked to plant resilience (Govindasamy et al., 2011; Loper et al., 2012). In hydroponics, PGPM species have been identified from the bacterial genera Pseudomonas, Bacillus, Enterobacter, Streptomyces, Gliocladium, and Trichoderma; many of which produce siderophores (Lee and Lee, 2015). It also has been demonstrated that siderophore production by a Chryseobacterium spp. alleviates iron starvation in tomato plants (Radzki et al., 2013). It is likely that other syntrophic or symbiotic relationships between plants and rhizoplane microbiomes exist, but as of now remain undiscovered or underutilized. As more PGPM's are discovered, operators may potentially integrate batch-culturing devices to facilitate the growth of PGPM's producing siderophores. Batch production could minimize industrially manufactured chelated iron input into the system, while aiding producers in ending the dispute over USDA organic certification for aquaponic and hydroponic produce (Biernbaum et al., 2016). At the 2017 National Organic Standards Board meeting no decision was made as to whether aquaponic or hydroponic crops could be certified organic under United States law.

Microbial iron use is a complicating factor in all environments with photosynthetic activity. In aquaponic systems, iron demand upstream of the hydroponic subsystem could induce a nutrient sink unless microbial micronutrient acquisition is considered in aquaponic system engineering. For example, an important design feature of aquaponics and recirculating aquaculture is solid waste decomposition, which induces anoxic or hypoxic conditions and facilitates methanogen growth when solid retention times (SRTs) are greater than 10 days (Suhr et al., 2015). System iron deficits may be compounded if solids management component SRT allows for methanogenic growth, since many methanogens and methanotrophs require iron in metallo-protein complexes to catalyze reactions (Speece, 1983; Glass and Orphan, 2012; Ettwig et al., 2016). Additionally, iron is closely linked to the nitrogen cycle; a critical nutrient cycle to manage for both fish and plant growth (Klotz and Stein, 2008; Timmons and Ebeling, 2013; Glass, 2015). In some systems, such as the ocean or recirculating aquaculture system solids digesters, the link between iron and nitrogen is significant, as denitrification pathways found in heterotrophic microorganisms constitute one of the largest group of iron dependent metabolic pathways (van Rijn et al., 2006; Glass et al., 2015). Careful consideration of solids management and microbial nitrification is essential to iron management in aquaponics, if siderophore-producing PGPM are to successfully mitigate chlorosis.

\section{THE MICROBIAL FUTURE OF AQUAPONICS}

Integrating PGPMs into aquaponic system design has the potential to alleviate micronutrient fluctuations and phytopathogen blooms in the hydroponic subsystem of an aquaponic system. PGPM use in aquaponic process engineering may maintain optimal plant production with lower nutrient concentrations than those found in a typical commercial hydroponic system, thereby reducing the incidence of disease, and abiotic inhibition of plant nutrient uptake (Mill et al., 1996; Rakocy et al., 1997; Lee and Lee, 2015). These operational conditions also may be maintained in a hydroponic system, but without fish feed and feces serving as the basis of plant growth substrate, external costs and maintenance increase (Villarroel et al., 2011). Typically, the aquaponic system operator supplements iron, calcium, and potassium, but solid waste re-mineralization reduces supplementation cost compared to stand alone hydroponic systems (Rakocy et al., 2006; Bittsanszky et al., 2016). Raising Tilapia (Oreochromis niloticus L.) at low stocking densities was found to reduce nutrient costs incurred in hydroponic strawberry production, however, PGPM growth was not considered (Villarroel et al., 2011). These results and those from other studies (e.g., Schmautz et al., 2016), suggest that fish effluent could serve as primary growth media for hydroponic subsystems, but supplemental nutrients may be needed depending on the stocking density of the fish, plant crop grown, and presence of active PGPM.

Besides PGPM research, there are a number of additional areas where microbial-based research could benefit aquaponic production. For example, aquaponic practitioners must carefully balance the $\mathrm{pH}$ requirements of fish, nitrifying microorganisms, and plants by identifying a mean $\mathrm{pH}$ that facilitates biological growth throughout all components, even if it is not optimal for any one component. Typically this means aquaponic operation at a $\mathrm{pH}$ of 7.0, whereas plants grown hydroponically prefer a lower pH, from 5.5 to 6.5 (Rakocy et al., 2006). However, $\mathrm{pH}$ balancing does not follow a concrete rule for operation, as a review of aquaponic crop production (Tyson et al., 2011) suggests normal total crop yields may be obtained at $\mathrm{pH}$ levels above those recommended for traditional production. Recent research into nitrifying microorganisms suggests that certain species of nitrite-oxidizing bacteria become more competitive at lower pH levels (Hüpeden et al., 2016), presenting the opportunity for further research into operating aquaponic systems at a lower $\mathrm{pH}$ and allowing $\mathrm{pH}$ to be optimized for specific plant or PGPM growth. There is also evidence of overlap between fish gut microbiomes and rhizosphere microbiomes 
(Hacquard et al., 2015), which could indicate microbial-based health benefits of fish-plant co-cultivation and an opportunity to use PGPM manipulation to benefit both plant and fish growth. Root-associated microorganisms are also known to influence plant phenology, such as flowering time (Wagner et al., 2014; Pérez-Jaramillo et al., 2016), and thus, in theory, could be used to manipulate desired plant biological characteristics in controlled settings such as those found in aquaponics. Finally, a recent model argues that a shift from continuous recirculation to decoupling aquaponic system components could lower incidences of nutrient supplementation and allow for PGPM growth planned into initial aquaponic system design (Goddek et al., 2016). Decoupling aquaponic components could free greater quantities of allochthonous iron and micronutrients from solid waste, while also sustaining crop growth during emergency or routine maintenance, but further research in this area and all those mentioned above is needed.

Despite the benefits of these integrated agricultural systems, achieving economic sustainability of aquaponic systems remains a challenge, primarily due to high capital expenses during system construction (Engle, 2015) and the need to reduce recurrent operational expenses (Love et al., 2015a). Microbial ecological theories governing nutrient cycling, host interactions, and community assembly underpin plant growth and health in aquaponic/hydroponic systems (Blancheton et al., 2013). Testing, understanding, and applying these theories to improve system design is crucial as global protein demands are becoming ever more reliant upon aquaculture/aquaponic systems, and many are turning to aquaponics to serve food deserts in cities and decrease agricultural water usage (Blidariu and Grozea, 2011; Goodman, 2011; FAO, 2014). We advocate that one "moon shot" research area for microbiologists should be enhancing sustainable agricultural systems, as this area presents opportunities to

\section{REFERENCES}

Akiyama, K., Matsuzaki, K., and Hayashi, H. (2005). Plant sesquiterpenes induce hyphal branching in arbuscular mycorrhizal fungi. Nature 435, 824-827. doi: $10.1038 /$ nature 03608

Andrews, S. C., Robinson, A. K., and Rodriguez-Quinones, F. (2003). Bacterial iron homeostasis. FEMS Microbiol. Rev. 27, 215-237. doi: 10.1016/S0168-6445(03) 00055-X

Barazani, O., and Baldwin, I. T. (2013). "A mixed bag: the plant growthpromoting Sebacina vermifera impairs defense mechanisms against herbivores," in Piriformospora Indica, eds A. Varma, G. Kost, and R. Oelmüller (Berlin: Springer-Verlag), 251-261. doi: 10.1007/978-3-642-33802-1

Barton, L. L., Johnson, G. V., and Schito, K. (1996). Siderophore-mediated iron metabolism in growth and nitrogen fixation by alfalfa nodulated with Rhizobium meliloti. J. Plant Nutr. 19, 1201-1210. doi: 10.1080/ 01904169609365191

Beijerinck, M. (1888). Die bacterien der papilionaceenknoellchen. Bot. Zeitung 46, 725-804.

Biernbaum, J., Chapman, D., Lam, T., Lamendella, A., Sideman, E., and Welsch, S. (2016). National Organic Standards Board (NOSB) Hydroponic and Aquaponic Task Force Report. Available at: https://www.ams.usda.gov/sites/default/files/ media/2016\%20Hydroponic\%20Task\%20Force\%20Report.PDF

Bittsanszky, A., Uzinger, N., Gyulai, G., Mathis, A., Junge, R., Villarroel, M., et al. (2016). Nutrient supply of plants in aquaponic systems. Ecocycles 2, 17-20. doi: 10.19040/ecocycles.v2i2.57

Blancheton, J. P., Attramadal, K. J. K., Michaud, L., d'Orbcastel, E. R., and Vadstein, O. (2013). Insight into bacterial population in aquaculture systems decentralize the food production system, simplifies agricultural logistics by decreasing distance to market, and enables more food safety and quality controls than traditional agriculture, while positively impacting local economies.

\section{AUTHOR CONTRIBUTIONS}

$\mathrm{RB}$ and JB developed and outlined the initial concept for the perspective. $\mathrm{RB}$ was the primary author and lead on writing and editing. BO, JB, and OS-V contributed ideas and writing for particular sections relating to their expertise, and all were involved in writing and editing of manuscript drafts. $\mathrm{RN}$ contributed to the initial outline and perspective focus, was involved in writing and editing of all manuscript stages, and was the primary source of funding for the project.

\section{FUNDING}

Funding for the work was provided by start-up laboratory funds to $\mathrm{RN}$ through the School of Freshwater Sciences, University of Wisconsin-Milwaukee, and a University of WisconsinMilwaukee Distinguished Dissertation Fellowship to RB.

\section{ACKNOWLEDGMENTS}

The authors thank Dave Love for providing insightful comments on previous versions of this manuscript and to all members of the Binkowski Lab for their many years of discussing aquaculture/aquaponic technology and providing access to a working aquaculture facility.

and its implication. Aquac. Eng. 53, 30-39. doi: 10.1016/j.aquaeng.2012. 11.009

Blidariu, F., and Grozea, A. (2011). Increasing the economical efficiency and sustainability of indoor fish farming by means of aquaponics. Anim. Sci. Biotechnol. 44, 1-8.

Boiteau, R., Hawco, N., Mende, D., McIlvin, M., Sedwick, P. N., Saito, M. A., et al. (2016). Siderophore-based adaptations to iron scarcity across major biomes of the eastern Pacific. Proc. Natl. Acad. Sci. U.S.A. 113, 14237-14242. doi: 10.1073/ pnas. 1608594113

Brear, E. M., Day, D. A., and Smith, P. M. C. (2013). Iron: an essential micronutrient for the legume-rhizobium symbiosis. Front. Plant Sci. 4:359. doi: 10.3389/fpls. 2013.00359

Carini, P., Marsden, P. J., Leff, J. W., Morgan, E. E., Strickland, M. S., and Fierer, N. (2016). Relic DNA is abundant in soil and obscures estimates of soil microbial diversity. Nat. Microbiol. 2:16242. doi: 10.1038/nmicrobiol.2016.242

Coleman-Derr, D., and Tringe, S. G. (2014). Building the crops of tomorrow: advantages of symbiont-based approaches to improving abiotic stress tolerance. Front. Microbiol. 5:283. doi: 10.3389/fmicb.2014.00283

da Silva Cerozi, B., and Fitzsimmons, K. (2016). Use of Bacillus spp. to enhance phosphorus availability and serve as a plant growth promoter in aquaponics systems. Sci. Hortic. 211, 277-282. doi: 10.1016/j.scienta.2016. 09.005

Danaher, J. J., Shultz, R. C., Rakocy, J. E., and Bailey, D. S. (2013). Alternative solids removal for warm water recirculating raft aquaponic systems. J. World Aquac. Soc. 44, 374-383. doi: 10.1111/jwas. 12040

Dessaux, Y., Grandclément, C., and Faure, D. (2016). Engineering the rhizosphere. Trends Plant Sci. 21, 266-278. doi: 10.1016/j.tplants.2016.01.002 
DeVries, F., and Wallenstein, M. D. (2017). Belowground connections underlying aboveground food production: a framework for optimising ecological connections in the rhizosphere. J. Ecol. 105, 913-920. doi: 10.1111/1365-2745. 12783

Dias, T., Dukes, A., and Antunes, P. M. (2014). Accounting for soil biotic effects on soil health and crop productivity in the design of crop rotations. J. Sci. Food Agric. 95, 447-454. doi: 10.1002/jsfa.6565

Engle, C. R. (2015). Economics of aquaponics. South. Reg. Aquac. Cent. 5006, 1-4.

Ettwig, K. F., Zhu, B., Speth, D., Keltjens, J. T., Jetten, M. S. M., and Kartal, B. (2016). Archaea catalyze iron-dependent anaerobic oxidation of methane. Proc. Natl. Acad. Sci. U.S.A. 113, 12792-12796. doi: 10.1073/pnas.1609534113

FAO (2014). The State of World Fisheries and Aquaculture. Available at: http://www.fao.org/3/a-i3720e.pdf

Fox, B. K., Tamaru, C. S., Hollyer, J., Castro, L. F., Fonseca, J. M., Jay-russell, M., et al. (2012). A preliminary study of microbial water quality related to food safety in recirculating aquaponic fish and vegetable production systems. Food Saf. Technol. 51, 1-11.

Genello, L., Fry, J. P., Frederick, J. A., Li, X., and Love, D. C. (2015). Fish in the classroom: a survey of the use of aquaponics in education. Eur. J. Heal. Biol. Educ. 4, 9-20. doi: 10.12973/ejhbe.2015.213p

Glass, J. B. (2015). Microbes that meddle with metals. Microbe Mag. 10, 197-202. doi: $10.1128 /$ microbe.10.197.1

Glass, J. B., Kretz, C. B., Ganesh, S., Ranjan, P., Seston, S. L., Buck, K. N., et al. (2015). Meta-omic signatures of microbial metal and nitrogen cycling in marine oxygen minimum zones. Front. Microbiol. 6:998. doi: 10.3389/fmicb.2015. 00998

Glass, J. B., and Orphan, V. J. (2012). Trace metal requirements for microbial enzymes involved in the production and consumption of methane and nitrous oxide. Front. Microbiol. 3:61. doi: 10.3389/fmicb.2012.00061

Goddek, S., Espinal, C., Delaide, B., Jijakli, M., Schmautz, Z., Wuertz, S., et al. (2016). Navigating towards decoupled aquaponic systems: a system dynamics design approach. Water 8:303. doi: 10.3390/w8070303

Goodman, E. R. (2011). Aquaponics: Community and Economic Development. Available at: http://hdl.handle.net/1721.1/67227

Govindasamy, V., Senthilkumar, M., Magheshwaran, V., Kumar, U., Bose, P., Sarma, V., et al. (2011). "Bacillus and Paenibacillus spp.: potential PGPR for sustainable agriculture," in Plant Growth and Health Promoting Bacteria, Vol. 18, ed. D. Maheshwari (Berlin: Springer-Verlag), 333-364. doi: 10.1007/978-3642-13612-2

Gravel, V., Martinez, C., Antoun, H., and Tweddell, R. J. (2006). Control of greenhouse tomato root rot [Pythium ultimum] in hydroponic systems, using plant-growth-promoting microorganisms. Can. J. Plant Pathol. 28, 475-483. doi: 10.1080/07060660609507322

Hacquard, S., Garrido-Oter, R., Gonzalez, A., Spaepen, S., Ackermann, G., Lebeis, S., et al. (2015). Review microbiota and host nutrition across plant and animal kingdoms. Cell Host Microbe 17, 603-616. doi: 10.1016/j.chom.2015. 04.009

Hart, E. R., Webb, J. B., Hollingsworth, C., and Danylchuk, A. J. (2014). Managing expectations for aquaponics in the classroom: enhancing academic learning and teaching an appreciation for aquatic resources. Fisheries 39, 525-530. doi: 10.1080/03632415.2014.966353

Höflich, G., Wiehe, W., and Hecht-Buchholz, C. (1995). Rhizosphere colonization of different crops with growth promoting Pseudomonas and Rhizobium bacteria. Microbiol. Res. 150, 139-147. doi: 10.1016/S0944-5013(11)80048-0

Hu, Z., Lee, J. W., Chandran, K., Kim, S., Brotto, A. C., and Khanal, S. K. (2015). Effect of plant species on nitrogen recovery in aquaponics. Bioresour. Technol. 188, 92-98. doi: 10.1016/j.biortech.2015.01.013

Hüpeden, J., Wegen, S., Off, S., Lucker, S., Bedarf, Y., Daims, H., et al. (2016). Relative abundance of Nitrotoga spp. in a biofilter of a cold-freshwater aquaculture plant appears to be stimulated by slightly acidic pH. Appl. Environ. Microbiol. 82, 1838-1845. doi: 10.1128/AEM.03163-15

Ji, S. H., Gururani, M. A., and Chun, S. (2014). Isolation and characterization of plant growth promoting endophytic diazotrophic bacteria from Korean rice cultivars ?. Microbiol. Res. 169, 83-98. doi: 10.1016/j.micres.2013.06.003

Khalifa, A. Y. Z., Alsyeeh, A.-M., Almalki, M. A., and Saleh, F. A. (2016). Characterization of the plant growth promoting bacterium, Enterobacter cloacae MSR1, isolated from roots of non-nodulating Medicago sativa. Saudi J. Biol. Sci. 23, 79-86. doi: 10.1016/j.sjbs.2015.06.008
Kloepper, J. W., Leong, J., Teintze, M., and Schroth, M. N. (1980). Enhanced plant growth by siderophores produced by plant growth-promoting rhizobacteria. Nature 286, 885-886. doi: 10.1038/286885a0

Klotz, M. G., and Stein, L. Y. (2008). Nitrifier genomics and evolution of the nitrogen cycle. FEMS Microbiol. Lett. 278, 146-156. doi: 10.1111/j.1574-6968. 2007.00970.x

Krsek, M., and Wellington, E. M. H. (1999). Comparison of different methods for the isolation and purification of total community DNA from soil. J. Microbiol. Methods 39, 1-16. doi: 10.1016/S0167-7012(99)00093-7

Lee, S., and Lee, J. (2015). Beneficial bacteria and fungi in hydroponic systems: types and characteristics of hydroponic food production methods. Sci. Hortic. 195, 206-215. doi: 10.1016/j.scienta.2015.09.011

Lewis, W. M., Yopp, J. H., Schramm, H. L. J., and Brandeburg, A. M. (1978). Use of hydroponics to maintain quality of recirculated water in a fish culture system. Trans. Am. Fish. Soc. 107, 92-99. doi: 10.1577/1548-8659(1978)107<92: UOHTMQ $>2.0 . \mathrm{CO} ; 2$

Loper, J. E., and Buyer, J. S. (1991). Siderophore in microbial interaction on plant surface. Mol. Plant Microbe Interact. 4, 5-13. doi: 10.1094/MPMI-4-005

Loper, J. E., Hassan, K. A., Mavrodi, D. V., Davis, E. W., Lim, C. K., Shaffer, B. T., et al. (2012). Comparative genomics of plant-associated pseudomonas spp.: insights into diversity and inheritance of traits involved in multitrophic interactions. PLOS Genet. 8:e1002784. doi: 10.1371/journal.pgen.1002784

Loper, J. E., and Henkels, M. D. (1997). Availability of iron to Pseudomonas fluorescens in rhizosphere and bulk soil evaluated with an ice nucleation reporter gene. Appl. Environ. Microbiol. 63, 99-105.

Loper, J. E., and Henkels, M. D. (1999). Utilization of heterologous siderophores enhances levels of iron available to Pseudomonas putida in the rhizosphere. Appl. Environ. Microbiol. 65, 5357-5363.

Love, D. C., Fry, J. P., Li, X., Hill, E. S., Genello, L., Semmens, K., et al. (2015a). Commercial aquaponics production and profitability: findings from an international survey. Aquaculture 435, 67-74. doi: 10.1016/j.aquaculture.2014.09.023

Love, D. C., Uhl, M. S., and Genello, L. (2015b). Energy and water use of a smallscale raft aquaponics system in Baltimore, Maryland, United States. Aquac. Eng. 68, 19-27. doi: 10.1016/j.aquaeng.2015.07.003

Majeed, A., Abbasi, M. K., Hameed, S., Imran, A., and Rahim, N. (2015). Solation and characterization of plant growth-promoting rhizobacteria from wheat rhizosphere and their effect on plant growth promotion. Front. Microbiol. 6:198. doi: $10.3389 /$ fmicb. 2015.00198

Malusá, E., Sas-Paszt, L., and Ciesielska, J. (2012). Technologies for beneficial microorganisms Inocula used as Biofertilizers. Sci. World J. 2012, 1-12. doi: 10.1100/2012/491206

Marasco, R., Rolli, E., Ettoumi, B., Vigani, G., Mapelli, F., Borin, S., et al. (2012). A drought resistance-promoting microbiome is selected by root system under desert farming. PLOS ONE 7:e48479. doi: 10.1371/journal.pone.0048479

Martin-Laurent, F., Philippot, L., Hallet, S., Chaussod, R., Soulas, G., and Catroux, G. (2001). DNA extraction from soils: old bias for new microbial diversity analysis methods DNA extraction from soils: old bias for new microbial diversity analysis methods. Appl. Environ. Microbiol. 67, 2354-2359. doi: 10.1128/AEM.67.5.2354

Medina, M., Jayachandran, K., Bhat, M., and Deoraj, A. (2016). Assessing plant growth, water quality and economic effects from application of a plantbased aquafeed in a recirculating aquaponics system. Aquac. Int. 24, 415-427. doi: 10.1007/s10499-015-9934-3

Mendes, R., Kruijt, M., De Bruijn, I., Dekkers, E., van der Voort, M., Schneider, J. H. M., et al. (2011). Deciphering the rhizosphere microbiome for disease-suppressive bacteria. Science 332, 1097-1100. doi: $10.1126 /$ science. 1203980

Mill, H. A., Wolf, B., and Jones, J. B. (1996). Plant Analysis Handbook II. Atlanta, GA: Micro Macro Publishing.

Mueller, U. G., and Sachs, J. L. (2015). Engineering microbiomes to improve plant and animal health. Trends Microbiol. 23, 606-617. doi: 10.1016/j.tim.2015. 07.009

Naegel, L. C. A. (1977). Combined production of fish and plants in recirculating water. Aquaculture 10, 17-24. doi: 10.1016/0044-8486(77)90029-1

Neilands, J., and Leong, S. A. (1986). Siderophores in relation to plant growth and disease. Annu. Rev. Plant Physiol. 37, 187-208. doi: 10.1146/annurev.pp.37.060186.001155 
Nguyen, N. T., McInturf, S. A., and Mendoza-Cózatl, D. G. (2016). Hydroponics: a versatile system to study nutrient allocation and plant responses to nutrient availability and exposure to toxic elements. J. Vis. Exp. 113:e54317. doi: $10.3791 / 54317$

Pérez-Jaramillo, J. E., Mendes, R., and Raaijmakers, J. M. (2016). Impact of plant domestication on rhizosphere microbiome assembly and functions. Plant Mol. Biol. 90, 635-644. doi: 10.1007/s11103-0150337-7

Pii, Y., Mimmo, T., Tomasi, N., Terzano, R., Cesco, S., and Crecchio, C. (2015). Microbial interactions in the rhizosphere: beneficial influences of plant growthpromoting rhizobacteria on nutrient acquisition process. Biol. Fertil. Soils 51, 403-415. doi: 10.1007/s00374-015-0996-1

Radzki, W., Gutierrez Mañero, F. J., Algar, E., Lucas García, J. A., GarcíaVillaraco, A., and Ramos Solano, B. (2013). Bacterial siderophores efficiently provide iron to iron-starved tomato plants in hydroponics culture. Antonie Van Leeuwenhoek 104, 321-330. doi: 10.1007/s10482-013-9954-9

Rakocy, J. E. (2012). “Chapter 14: aquaponics-integrating fish and plant culture," in Aquaculture Production Systems, 1st Edn, ed. J. Tidwell (Hoboken, NJ: John Wiley \& Sons), 343-386.

Rakocy, J. E., Bailey, D. S., Shultz, K. A., and Cole, W. M. (1997). "Evaluation of a commercial-scale aquaponic unit for the production of tilapia and lettuce," in Tilapia Aquaculture: Proceedings of the Fourth International Symposium on Tilapia in Aquaculture, Orlando, FL. 357-372.

Rakocy, J. E., Bailey, D. S., Shultz, R. C., and Thoman, E. S. (2004). "Update on tilapia and vegetable production in the UVI aquaponic system. New dimensions on farmed tilapia," in Proceedings from the 6th International Symposium on Tilapia in Aquaculture (Manila, Philippines: International Symposium on Tilapia in Aquaculture), 1-15.

Rakocy, J. E., Masser, M. P., and Losordo, T. M. (2006). Recirculating aquaculture tank production systems: aquaponics- integrating fish and plant culture. South. Reg. Aquac. Cent. 454, 1-16.

Rakocy, J. E., Masser, M. P., and Losordo, T. M. (2012). Recirculating aquaculture tank production systems: aquaponics-integrating fish and plant culture. South. Reg. Aquac. Cent. 454, 1-16.

Rashid, S., Charles, T. C., and Glick, B. R. (2012). Isolation and characterization of new plant growth-promoting bacterial endophytes. Appl. Soil Ecol. 61, 217-224. doi: 10.1016/j.apsoil.2011.09.011

Roosta, H. R. (2014). Comparison of the vegetative growth, eco-physiological characteristics and mineral nutrient content of basil plants in different irrigation ratios of hydroponic:aquaponic solutions. J. Plant Nutr. 37, 1782-1803. doi: 10.1080/01904167.2014.890220

Rose, A. L., and Waite, T. D. (2002). Kinetic model for Fe(II) oxidation in seawater in the absence and presence of natural organic matter. Environ. Sci. Technol. 36, 433-444. doi: 10.1021/es0109242

Savka, M. A., Dessaux, Y., Gardener, B. B. M., Mondy, S., Kohler, P. R. A., de Bruijn, F. J., et al. (2013). "Engineering the rhizosphere: the 'Biased Rhizosphere ' concept the 'Biased Rhizosphere' concept and advances in the omics era to study bacterial competitiveness and persistence in the phytosphere," in Molecular Microbial Ecology of the Rhizosphere, 1st Edn, ed. F. J. de Bruijn (Hoboken, NJ: Wiley Blackwell), 1147-1161.

Schmautz, Z., Graber, A., Jaenicke, S., Goesmann, A., Junge, R., and Smits, T. H. M. (2017). Microbial diversity in different compartments of an aquaponics system. Arch. Microbiol. 199, 613-620. doi: 10.1007/s00203-016-1334-1

Schmautz, Z., Loeu, F., Liebisch, F., Graber, A., Mathis, A., Bulc, T. G., et al. (2016). Tomato productivity and quality in aquaponics: comparison of three hydroponic methods. Water 8, 1-21. doi: 10.3390/w8110533

Sheridan, C., Depuydt, P., de Ro, M., Petit, C., van Gysegem, E., Delaere, P., et al. (2016). microbial community dynamics and response to plant growth-promoting microorganisms in the rhizosphere of four common food crops cultivated in hydroponics. Microb. Ecol. 73, 378-393. doi: 10.1007/s00248-016-0855-0

Sneed, K., Allen, K., and Ellis, J. (1975). Fish farming and hydroponics. Aquac. Fish Farmer 2, 18-20.

Speece, R. E. (1983). Anaerobic biotechnology for industrial wastewater treatment. Environ. Sci. Technol. 17, 416A-427A. doi: 10.1021/es00115a725

Suhr, K. I., Letelier-Gordo, C. O., and Lund, I. (2015). Anaerobic digestion of solid waste in RAS: effect of reactor type on the biochemical acidogenic potential (BAP) and assessment of the biochemical methane potential (BMP) by a batch assay. Aquac. Eng. 65, 65-71. doi: 10.1016/j.aquaeng.2014. 12.005

Timmons, M. B., and Ebeling, J. M. (2013). Recirculating Aquaculture, 3rd Edn. Ithaca, NY: Ithaca Publishing Company.

Tyson, R. V., Treadwel, D. D., and Simonne, E. H. (2011). Opportunities and challenges to sustainability in aquaponic systems. Horttechnology 21, 6-13.

Utkhede, R. (2006). Increased growth and yield of hydroponically grown greenhouse tomato plants inoculated with arbuscular mycorrhizal fungi and Fusarium oxysporum f. sp. Radicis-lycopersici. BioControl 51, 393-400. doi: 10.1007/s10526-005-4243-9

van Dijk, J. P., Lagerwerf, A. J., van Eijk, H. G., and Leijnse, B. (1975). Iron metabolism in the tench (Tinca tinca L.). J. Comp. Physiol. 99, 321-330. doi: 10.1007/BF00710372

van Rijn, J., Tal, Y., and Schreier, H. J. (2006). Denitrification in recirculating systems: theory and applications. Aquac. Eng. 34, 364-376. doi: 10.1016/j.aquaeng.2005.04.004

Villarroel, M., Alvariño, J. M. R., and Duran, J. M. (2011). Aquaponics: integrating fish feeding rates and ion waste production for strawberry hydroponics. Spanish J. Agric. Res. 9, 537-545. doi: 10.5424/sjar/20110902-181-10

Wagner, M. R., Lundberg, D. S., Coleman-Derr, D., Tringe, S. G., Dangl, J. L., and Mitchell-Olds, T. (2014). Natural soil microbes alter flowering phenology and the intensity of selection on flowering time in a wild Arabidopsis relative. Ecol. Lett. 17, 717-726. doi: 10.1111/ele.12276

Waite, T. D. (2002). Challenges and opportunities in the use of iron in water and wastewater treatment. Rev. Environ. Sci. Biotechnol. 1, 9-15. doi: 10.1023/A: 1015131528247

Watanabe, T., Kiron, V., and Satoh, S. (1997). Trace minerals in fish nutrition. Aquaculture 151, 185-207. doi: 10.1016/S0044-8486(96)01503-7

Conflict of Interest Statement: The authors declare that the research was conducted in the absence of any commercial or financial relationships that could be construed as a potential conflict of interest.

Copyright (C) 2018 Bartelme, Oyserman, Blom, Sepulveda-Villet and Newton. This is an open-access article distributed under the terms of the Creative Commons Attribution License (CC BY). The use, distribution or reproduction in other forums is permitted, provided the original author(s) or licensor are credited and that the original publication in this journal is cited, in accordance with accepted academic practice. No use, distribution or reproduction is permitted which does not comply with these terms. 